\title{
Viscoelastic Materials
}

Rubber and other elastomers are highly deformable and thus conform well to surfaces, exhibit high coefficients of friction in many material pairings (rubber-rubber, rubber-asphalt, etc.), and are water and heat resistant. As a result, elastomers see widespread use in tires, belts, cables, adhesive layers, and many other areas of application.

The most important properties of elastomers are: (1) an extremely low elasticity modulus (about 1 to $10 \mathrm{MPa}$, i.e., four to five orders of magnitude lower than that of "normal" solids), (2) an extreme degree of deformability, and (3) internal energy dissipation (viscosity) during deformation. The root of these fundamental properties of elastomers lies in their structure. Elastomers consist of relatively weakly interacting polymer molecules. In the thermodynamic state of equilibrium, they are in a statistically favored coiled state. Upon application of a mechanical stress on the elastomer, the polymer molecules begin to uncoil. Removing the load from the elastomer causes the polymer molecules to relax once again into their coiled state. This structure explains both the high deformability and the internal friction, along with the associated time-dependent behavior, of elastomers.

Due to the high degree of deformations, elastomers very often exhibit non-linear mechanical behavior. However, for reasons of simplification, we will treat them here as linear viscoelastic materials. The treatment of non-linearities would extend beyond the scope of this book.

Chapter 8 is dedicated to contact problems between a rigid, rotationally symmetric indenter and a homogeneous, isotropic, linear viscoelastic half-space. Section 8.1 will provide some initial general information and definitions regarding the description and characterization of linear viscoelastic materials. Sections 8.2 and 8.3 are dedicated to the explicit solution of axially symmetric contact problems using the MDR and the functional equation method by Lee and Radok (1960). These solutions apply to incompressible elastomers. Compressible normal contacts are discussed in Sect. 8.4. Finally, Sect. 8.5 deals with fretting wear of elastomers. 


\subsection{General Information and Definitions on Viscoelastic Media}

\subsubsection{Time-Dependent Shear Modulus and Creep Function}

In a first-order approximation, elastomers can be viewed as incompressible media (Poisson's ratio $v \approx 0.5$ ). Therefore, the following consideration exclusively deals with the characterization of mechanical properties of elastomers for shear loads. Let us consider an elastomer block that is being acted upon by shear forces (Fig. 8.1). If it is rapidly deformed by the shear angle $\varepsilon_{0}{ }^{1}$, the stress initially rises to a high level $\sigma(0)$ and subsequently relaxes to a much lower value $\sigma(\infty)$ (Fig. 8.2). In elastomers, $\sigma(\infty)$ can be three to four orders of magnitudes smaller than $\sigma(0)$. The ratio

$$
G(t)=\frac{\sigma(t)}{\varepsilon_{0}}
$$

is called the time-dependent shear modulus. This function completely describes the mechanical properties of a material, assuming that the material exhibits a linear behavior.

Let us assume that the block is deformed according to an arbitrary function $\varepsilon(t)$. Any time-dependency $\varepsilon(t)$ can be represented as the sum of time-shifted step functions, as shown schematically in Fig. 8.3. In this diagram, an "elementary step function" at time $t^{\prime}$ has the amplitude $\mathrm{d} \varepsilon\left(t^{\prime}\right)=\dot{\varepsilon}\left(t^{\prime}\right) \mathrm{d} t^{\prime}$. Accordingly, its contribution to the stress is equal to $\mathrm{d} \sigma=G\left(t-t^{\prime}\right) \dot{\varepsilon}\left(t^{\prime}\right) \mathrm{d} t^{\prime}$ and the total stress at every point

Fig. 8.1 Shear deformation of a rubber block

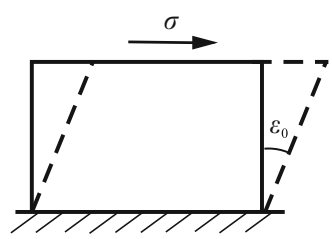

a

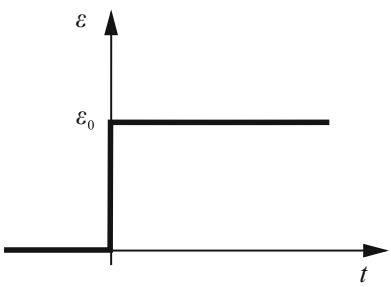

b

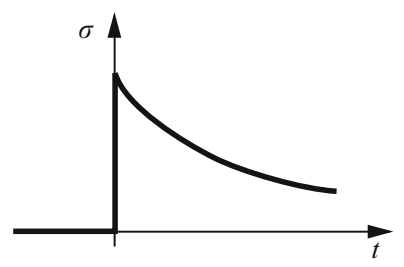

Fig. 8.2 If a rubber block is rapidly deformed by $\varepsilon_{0}$ at time $t=0$, then the stresses rise to an initial high level and subsequently relax slowly to a much lower value

\footnotetext{
${ }^{1}$ We emphasize that the shear angle $\varepsilon$ is equal to twice the shear component of the deformation tensor.
} 
Fig. 8.3 Representation of a time-dependent function as the superposition of several shifted step functions

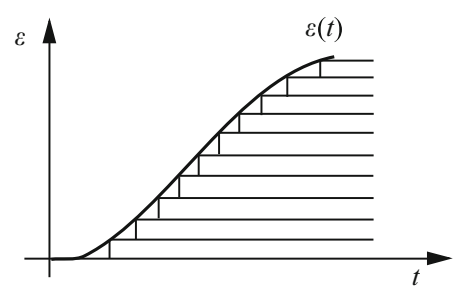

in time is, therefore, calculated as:

$$
\sigma(t)=\int_{-\infty}^{t} G\left(t-t^{\prime}\right) \dot{\varepsilon}\left(t^{\prime}\right) \mathrm{d} t^{\prime}
$$

Alternatively, the block can be effected by a sudden tangential force. After an instantaneous reaction, the shear angle will change with time. The time-dependent shear angle related to the stress is called the creep function, $\Phi(t)$ :

$$
\Phi(t)=\frac{\varepsilon(t)}{\sigma_{0}} .
$$

Using the creep function, we can write the relationship between the stress and strain similarly to (8.2):

$$
\varepsilon(t)=\int_{-\infty}^{t} \Phi\left(t-t^{\prime}\right) \dot{\sigma}\left(t^{\prime}\right) \mathrm{d} t^{\prime} .
$$

It can be shown that representations (8.1) and (8.4) are equivalent, i.e., for any time-dependent shear modulus a corresponding creep function can be determined and vice-versa.

From the definitions of the time-dependent shear modulus and the creep functions, the following identities can be deducted:

$$
\int_{-\infty}^{t} G\left(t-t^{\prime}\right) \dot{\Phi}\left(t^{\prime}\right) \mathrm{d} t^{\prime}=1
$$

and

$$
\int_{-\infty}^{t} \Phi\left(t-t^{\prime}\right) \dot{G}\left(t^{\prime}\right) \mathrm{d} t^{\prime}=1 .
$$

Technically, every elastomer also has a material law for the trace (hydrostatic) components of the stress and deformation tensor corresponding to a second creep function for the deformation response to hydrostatic pressure. However, as previously mentioned, elastomers can usually be considered incompressible. Therefore, 
this secondary creep function is usually neglected. Taking both creep functions into account also makes the analytical treatment of viscoelastic contact problems far more difficult, as demonstrated by Vandamme and Ulm (2006). With these facts in mind, in this chapter we will generally limit ourselves to solutions for an incompressible half-space. The consideration of the compressible case is touched upon in Sect. 8.4.

\subsubsection{Complex, Dynamic Shear Modulus}

If $\varepsilon(t)$ changes according to the harmonic function

$$
\varepsilon(t)=\tilde{\varepsilon} \cos (\omega t),
$$

then after the transient time, there will also be a periodic change in stress at the same frequency $\omega$. The relationship between the change in deformation and the stress can be represented quite simply when the real function $\cos (\omega t)$ is presented as the sum of two complex exponential functions:

$$
\cos (\omega t)=\frac{1}{2}\left(e^{i \omega t}+e^{-i \omega t}\right) .
$$

Due to the principle of superposition, one can initially calculate the stresses resulting from the complex oscillations

$$
\varepsilon(t)=\tilde{\varepsilon} e^{i \omega t} \quad \text { and } \quad \varepsilon(t)=\tilde{\varepsilon} e^{-i \omega t}
$$

and sum them up. If we insert $\varepsilon(t)=\tilde{\varepsilon} e^{i \omega t}$ into (8.2), then we obtain

$$
\sigma(t)=\int_{-\infty}^{t} G\left(t-t^{\prime}\right) i \omega \tilde{\varepsilon} e^{i \omega t^{\prime}} \mathrm{d} t^{\prime}=i \omega \tilde{\varepsilon} e^{i \omega t} \int_{0}^{\infty} G(\xi) e^{-i \omega \xi} \mathrm{d} \xi
$$

for the stress, where we substituted $\xi=t-t^{\prime}$. This relation can also be written in the form:

$$
\sigma(t)=\hat{G}(\omega) \tilde{\varepsilon} e^{i \omega t}=\hat{G}(\omega) \varepsilon(t) .
$$

The coefficient of proportionality

$$
\hat{G}(\omega)=i \omega \int_{0}^{\infty} G(\xi) e^{-i \omega \xi} \mathrm{d} \xi
$$

is called the complex shear modulus. Its real part $G^{\prime}(\omega)=\operatorname{Re} \hat{G}(\omega)$ is called the storage modulus, and its imaginary part $G^{\prime \prime}(\omega)=\operatorname{Im} \hat{G}(\omega)$ is referred to as the loss modulus. 


\subsubsection{Rheological Models}

The properties of viscoelastic media are frequently presented in the form of rheological models. The two fundamental elements of these models are:

(a) A linear-elastic body. For an ideally elastic body, the shear deformation follows Hooke's law: $\sigma=G \varepsilon$. In this case, the complex modulus only has a real part which is equal to $G$ :

$$
\hat{G}=G
$$

(b) A linear viscous fluid. For which the following is valid: (see Fig. 8.4):

$$
\sigma=\eta \frac{\mathrm{d} v}{\mathrm{~d} z}
$$

For a periodic displacement $\hat{u}(l, t)=u_{0} e^{i \omega t}$ we get:

$$
\hat{\sigma}(t)=\left.\eta \frac{\mathrm{d} v}{\mathrm{~d} z}\right|_{z=l}=\eta \frac{\hat{v}(t)}{l}=\eta i \omega \frac{u_{0}}{l} e^{i \omega t}=i \omega \eta \hat{\varepsilon}(t) .
$$

In this case the complex modulus

$$
\hat{G}(\omega)=i \omega \eta
$$

only has an imaginary part: $\operatorname{Re} \hat{G}=0, \operatorname{Im} \hat{G}=\omega \eta$.

These two elements enable the "construction" of different media. Although we will refer to the rheological models as "springs" and "dampers", in actuality we mean the corresponding quantities per unit volume of the medium. In this case, it should be noted that the tangential stiffness equals the shear modulus and the damping coefficient equals the dynamic viscosity. From now on we will use these continuum mechanical terms of moduli and viscosities rather than those of values of stiffness and damping coefficients.

\subsubsection{Kelvin Medium}

One of the most frequently used rheological models is the Kelvin model, consisting of a spring (shear modulus $G$ ), connected in parallel with a damper (viscosity $\eta$ ). The complex shear modulus of this medium is equal to

$$
\hat{G}=G+i \omega \eta \text {. }
$$

Fig. 8.4 Uniform shear flow of a linear viscous fluid

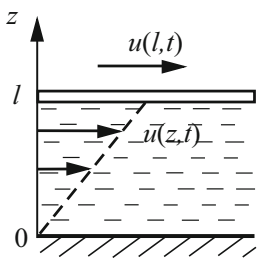


If at time $t=0$ a constant stress $\sigma_{0}$ is applied to the Kelvin element, the following is valid:

$$
G \varepsilon+\eta \dot{\varepsilon}=\sigma_{0} .
$$

The shear angle is time-dependent according to

$$
\varepsilon=\frac{\sigma_{0}}{G}\left(1-e^{-t / \tau}\right)
$$

with $\tau=\eta / G$. The creep function of this medium is, therefore, equal to:

$$
\Phi(t)=\frac{\varepsilon(t)}{\sigma_{0}}=\frac{1}{G}\left(1-e^{-t / \tau}\right) .
$$

\subsubsection{Maxwell Medium}

An important component of many rheological models is the Maxwell element, consisting of a spring connected in series with a linear viscous damper.

The complex moduli of the spring and damper are $G$ and $i \eta \omega$. Due to the serial arrangement, we obtain:

$$
\hat{G}_{\text {Maxwell }}=\frac{G \cdot i \eta \omega}{G+i \eta \omega}=\frac{G \cdot i \eta \omega}{(G+i \eta \omega)} \frac{(G-i \eta \omega)}{(G-i \eta \omega)}=\frac{G\left(i \eta \omega G+(\eta \omega)^{2}\right)}{G^{2}+(\eta \omega)^{2}}
$$

for the total modulus.

The storage and loss moduli are:

$$
G_{\text {Maxwell }}^{\prime}=\frac{G(\eta \omega)^{2}}{G^{2}+(\eta \omega)^{2}}, \quad G_{\text {Maxwell }}^{\prime \prime}=\frac{\eta \omega G^{2}}{G^{2}+(\eta \omega)^{2}} .
$$

By introducing the quantity

$$
\tau=\eta / G,
$$

(8.22) can also be presented in the form:

$$
G_{\text {Maxwell }}^{\prime}=G \frac{(\omega \tau)^{2}}{1+(\omega \tau)^{2}}, \quad G_{\text {Maxwell }}^{\prime \prime}=G \frac{\omega \tau}{1+(\omega \tau)^{2}} .
$$

The quantity $\tau$ has the dimension time and is called the relaxation time.

Let us examine the stress relaxation in a medium which is described by a Maxwell element. We use the terms introduced in Fig. 8.5. The stress acting on the connection point between the spring and the damper is equal to $-G\left(\varepsilon-\varepsilon_{1}\right)+\eta \dot{\varepsilon}_{1}$. Because the connection point is massless, the stress must cancel out: $-G\left(\varepsilon-\varepsilon_{1}\right)+\eta \dot{\varepsilon}_{1}=0$. By dividing this equation by $G$ and inserting (8.23), we can write the equation as:

$$
\tau \dot{\varepsilon}_{1}+\varepsilon_{1}=\varepsilon .
$$


Fig. 8.5 Maxwell element

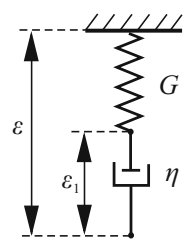

If at time $t=0$ the material is suddenly deformed by $\varepsilon_{0}$, then for every point in time $t>0$ we get

$$
\tau \dot{\varepsilon}_{1}+\varepsilon_{1}=\varepsilon_{0},
$$

with the initial condition $\varepsilon_{1}(0)=0$. The solution to this equation with the stated initial condition is:

$$
\varepsilon_{1}=\varepsilon_{0}\left(1-e^{-t / \tau}\right)
$$

The stress is:

$$
\sigma=G\left(\varepsilon_{0}-\varepsilon_{1}\right)=G \varepsilon_{0} e^{-t / \tau}
$$

The stress decays exponentially with the characteristic time $\tau$. Therefore, the timedependent shear modulus in this case equals:

$$
G(t)=G e^{-t / \tau}
$$

\subsubsection{The Standard Solid Model of Rubber}

The following model (Fig. 8.6) is the simplest spring-damper model to give a qualitatively correct representation of the most important dynamic properties of rubber during periodic loading.

Since we are dealing with a parallel arrangement of a linear-elastic spring and a Maxwell element, we can immediately write:

$$
G^{\prime}=G_{1}+G_{2} \frac{(\omega \tau)^{2}}{1+(\omega \tau)^{2}}, \quad G^{\prime \prime}=G_{2} \frac{\omega \tau}{1+(\omega \tau)^{2}},
$$

with $\tau=\eta / G_{2}$. The frequency-dependency of the moduli is presented double logarithmically for the case $G_{2} / G_{1}=1000$ in Fig. 8.7.

At low frequencies $\omega<G_{1} / \eta$ (quasi-static loading) the modulus approaches $G_{1}$. At very high frequencies $\omega>G_{2} / \eta$ it approaches $G_{1}+G_{2} \gg G_{1}$. This means that for very slow loading, rubber is soft, while for rapid loading it is stiff. In the

Fig. 8.6 A simple rheological model of rubber (standard solid model)

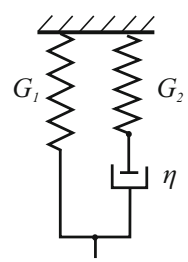


Fig. 8.7 Real and imaginary parts of the dynamic modulus for the rheological model shown in Fig. 8.6 with $G_{2} / G_{1}=1000$

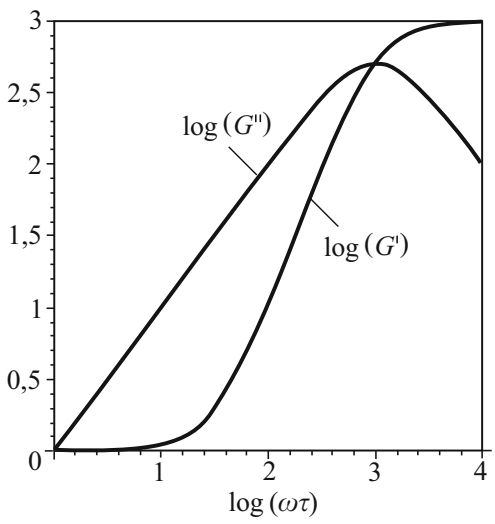

intermediate range, the imaginary part is dominant: $G^{\prime \prime}(\omega) \approx \eta \omega$, meaning that the medium behaves like a viscous fluid during periodic loading.

Once again, since we are dealing with a parallel arrangement of a linear-elastic spring and a Maxwell element, we can immediately write:

$$
\sigma(t)=\varepsilon_{0}\left(G_{1}+G_{2} e^{-t / \tau}\right)
$$

The normalized stress, which we have called the time-dependent modulus, can be obtained by dividing this equation by $\varepsilon_{0}$ :

$$
G(t)=\sigma / \varepsilon_{0}=\left(G_{1}+G_{2} e^{-t / \tau}\right) .
$$

It relaxes exponentially from the value $G_{0}=G_{1}+G_{2}$ for $t=0$ to the value $G_{\infty}=G_{1}$ for $t \rightarrow \infty$.

\subsubsection{Summary: Creep Function, Relaxation Function, and Complex Shear Modulus for the Four Most Common Viscous/Viscoelastic Material Models}

The creep functions, relaxation functions, and complex shear moduli of the three most frequently used simple viscoelastic material models (standard solid, Maxwell solid, Kelvin solid) are presented in normalized form in Table 8.1. The linear viscous half-space results as the limiting case of the Maxwell element for $\tau \rightarrow 0$ and as the limiting case of the Kelvin-Voigt element for $\tau \rightarrow \infty$. Figure 8.8 shows phase diagrams of the complex shear modulus of the standard element.

Generalized Maxwell or Kelvin solids are frequently used to model more complex material behavior. These models can be given in the form of Prony series with varying relaxation times. However, since an exact analytical treatment of such material behavior is usually impossible, we will restrict our consideration to the simple models presented previously in this chapter. 


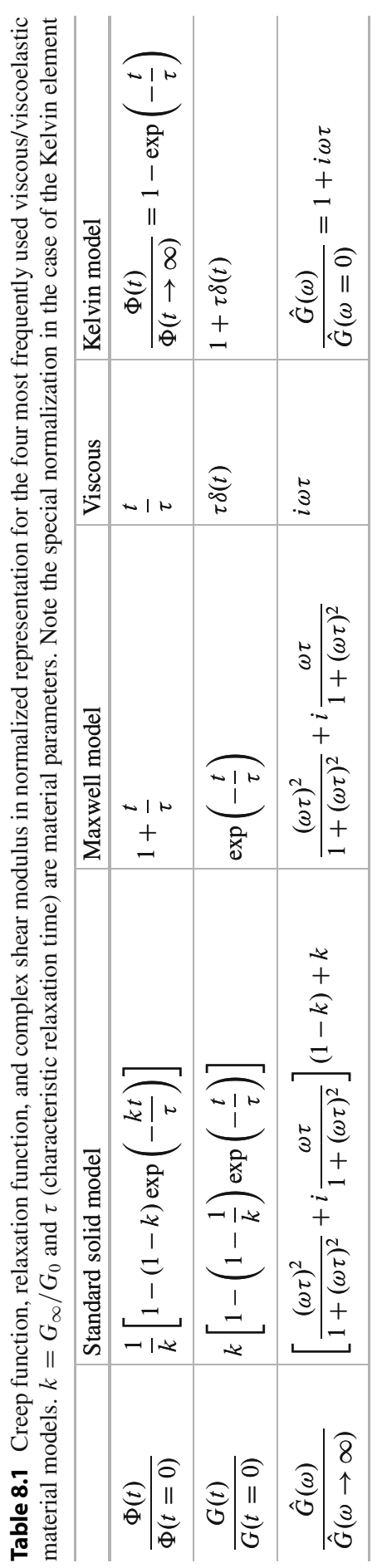


Fig. 8.8 Phase diagram of the complex shear modulus of a standard solid model for different values of $k=G_{\infty} / G_{0}$ with the parameter $\omega \tau$. The thin solid lines represent the limiting cases of the Maxwell and Kelvin models (vertical). The maximum of the normalized loss modulus lies at $\omega \tau=1$

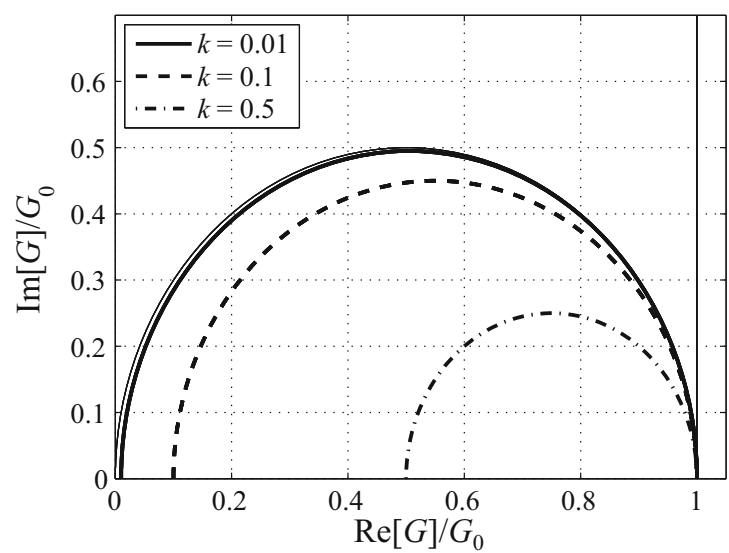

\subsubsection{Application of the MDR to Viscoelastic Media}

If the indentation velocity of an elastomer during a dynamic loading is below the lowest speed of wave propagation (which is defined by the lowest relevant modulus) then the contact can be regarded as quasi-static. If this condition is met and an area of an elastomer is excited with the angular frequency $\omega$, there is a linear relationship between stress and deformation and, consequently, between force and displacement. The medium can be viewed as an elastic body with the effective shear modulus $G(\omega)$. All principles that are valid for a purely elastic body must also be valid for the harmonically excited viscoelastic medium. Therefore, elastomers can also be described using the MDR as presented in Chaps. 2 and 4. The only difference to the elastic contact is that the effective elasticity modulus is now a function of frequency. For incompressible media $(v=1 / 2)$, the following equation is valid for springs of the effective Winkler foundation:

$$
\Delta k_{z}(\omega)=E^{*}(\omega) \Delta x=\frac{E(\omega)}{1-v^{2}} \Delta x=\frac{2 G(\omega)}{1-v} \Delta x \approx 4 G(\omega) \Delta x .
$$

The stiffness of an individual "spring" of the Winkler foundation is four times the shear modulus multiplied by the discretization step size. For a harmonic excitation of the one-dimensional equivalent system, we obtain the spring force:

$$
\Delta F_{N}(x, \omega)=\frac{2 G(\omega)}{1-v} \Delta x \cdot w_{1 D}(x, \omega) \approx 4 G(\omega) \Delta x \cdot w_{1 D}(x, \omega) .
$$


The inverse transformation into the time domain gives the force as an explicit function of time:

$$
\begin{aligned}
\Delta F_{N}(x, t) & =\frac{2}{1-v} \Delta x \int_{-\infty}^{t} G\left(t-t^{\prime}\right) \dot{w}_{1 D}\left(x, t^{\prime}\right) \mathrm{d} t^{\prime} \\
& \approx 4 \Delta x \int_{-\infty}^{t} G\left(t-t^{\prime}\right) \dot{w}_{1 D}\left(x, t^{\prime}\right) \mathrm{d} t^{\prime} .
\end{aligned}
$$

For tangential contacts, the tangential stiffness of the springs of the equivalent onedimensional MDR foundation must be defined in accordance with (4.21):

$$
\Delta k_{x}=G^{*}(\omega) \Delta x=\frac{4 G(\omega)}{2-v} \Delta x \approx \frac{8}{3} G(\omega) \Delta x .
$$

The corresponding force in the time domain is:

$$
\begin{aligned}
\Delta F_{x}(t) & =\frac{4}{2-v} \Delta x \int_{-\infty}^{t} G\left(t-t^{\prime}\right) \dot{u}_{1 D}\left(t^{\prime}\right) \mathrm{d} t^{\prime} \\
& \approx \frac{8}{3} \Delta x \int_{-\infty}^{t} G\left(t-t^{\prime}\right) \dot{u}_{1 D}\left(t^{\prime}\right) \mathrm{d} t^{\prime} .
\end{aligned}
$$

The formal mathematical proof of this method is based on the method of functional equations by Radok (1957) and was presented by Popov and Heß (2015).

Using rheological models instead of the integral representations (8.35) and (8.37), the forces in the two fundamental elements of elastic bodies and fluids are then given by

$$
\Delta F_{N}=4 G w_{1 D} \Delta x \text { and } \Delta F_{N}=4 \eta \dot{w}_{1 D} \Delta x
$$

for the normal force, and by

$$
\Delta F_{x}=\frac{8}{3} G u_{1 D} \Delta x \quad \text { and } \quad \Delta F_{x}=\frac{8}{3} \eta \dot{u}_{1 D} \Delta x
$$

for the tangential force.

In summary, here are the most important steps of applying the MDR to viscoelastic contacts of non-compressible media: 
I. The three-dimensional viscoelastic body is substituted by a onedimensional Winkler foundation consisting of rheological models, which is defined by the following functions of force:

$$
\begin{aligned}
\Delta F_{N}(x, t) & =\frac{2}{1-v} \Delta x \int_{-\infty}^{t} G\left(t-t^{\prime}\right) \dot{w}_{1 D}\left(x, t^{\prime}\right) \mathrm{d} t^{\prime} \\
& \approx 4 \Delta x \int_{-\infty}^{t} G\left(t-t^{\prime}\right) \dot{w}_{1 D}\left(x, t^{\prime}\right) \mathrm{d} t^{\prime}, \\
\Delta F_{x}(t)= & \frac{4}{2-v} \Delta x \int_{-\infty}^{t} G\left(t-t^{\prime}\right) \dot{u}_{1 D}\left(t^{\prime}\right) \mathrm{d} t^{\prime} \\
\approx & \frac{8}{3} \Delta x \int_{-\infty}^{t} G\left(t-t^{\prime}\right) \dot{u}_{1 D}\left(t^{\prime}\right) \mathrm{d} t^{\prime},
\end{aligned}
$$

or alternatively, is constructed from the usual rheological models of springs (stiffness $k$ ) and dampers (damping coefficients $\alpha$ ) in accordance with:

$$
\begin{aligned}
& \Delta k_{z}=4 G \Delta x \quad \text { and } \quad \Delta \alpha=4 \eta \Delta x \\
& \Delta k_{x}=\frac{8}{3} G \Delta x \quad \text { and } \quad \Delta \alpha_{x}=\frac{8}{3} \eta \Delta x .
\end{aligned}
$$

II. The three-dimensional profile $\tilde{z}=f(r)$ is transformed into a onedimensional profile $g(x)$ according to:

$$
g(x)=|x| \int_{0}^{|x|} \frac{f^{\prime}(r)}{\sqrt{x^{2}-r^{2}}} \mathrm{~d} r .
$$

III. The one-dimensional profile according to (8.44) is now pressed into the viscoelastic foundation according to (8.40)-(8.43). The relationships between the normal force, the indentation depth, and the contact radius resulting from the one-dimensional model corresponds exactly to the ones of the original three-dimensional problem at every point in time and independent of the loading history.

IV. The stress distribution can, if necessary, also be calculated using an equation given in Chap. 2 (2.13) and the deformation of the medium (including the area outside the contact) also with an (2.14) from Chap. 2. 


\subsubsection{Description of Elastomers by Radok (1957)}

By substituting $t^{\prime}=t-\xi$ and expanding (8.2) to powers of $\xi$, (8.2) can be rewritten in the following differential form:

$$
\sigma(t)=\int_{0}^{\infty} G(\xi) \dot{\varepsilon}(t-\xi) \mathrm{d} \xi=\sum_{n=1}^{\infty} \frac{\mathrm{d}^{n} \varepsilon(t)}{\mathrm{d} t^{n}} \int_{0}^{\infty} \frac{(-1)^{n}}{n !} \xi^{n} G(\xi) \mathrm{d} \xi .
$$

Similarly, (8.4) can be written in the form:

$$
\varepsilon(t)=\sum_{n=1}^{\infty} \frac{\mathrm{d}^{n} \sigma(t)}{\mathrm{d} t_{n}} \int_{0}^{\infty} \frac{(-1)^{n}}{n !} \xi^{n} \Phi(\xi) \mathrm{d} \xi .
$$

Equation (8.18) for the Kelvin model is a simple example of the series expansion (8.45). For the case of the Kelvin model, only the terms of the order $n=0$ and $n=1$ appear.

Radok (1957) noted that representations (8.45) and (8.46) were merely special cases of a more general representation

$$
P\left[s_{i j}(t)\right]=Q\left[e_{i j}(t)\right],
$$

where $s_{i j}$ and $e_{i j}$ denote the traceless components of the stress tensor and deformation tensor in Cartesian coordinates. $P$ and $Q$ are the linear differential operators defined as:

$$
\begin{gathered}
P:=\sum_{k=0}^{\infty} p_{k} \frac{\mathrm{d}^{k}}{\mathrm{~d} t^{k}}, \\
Q:=\sum_{k=0}^{\infty} q_{k} \frac{\mathrm{d}^{k}}{\mathrm{~d} t^{k}} .
\end{gathered}
$$

In the purely elastic case, these are zero-order operators with

$$
\begin{gathered}
\frac{q_{0}}{p_{0}}=2 G, \\
q_{k}=0, \quad p_{k}=0, \quad k>0,
\end{gathered}
$$

and the shear modulus $G$. It is easy to see that the two operators are simply indeterminate, yet this can be easily resolved by an appropriate normalization. The identities (8.5) and (8.6) can be written in the form

$$
\begin{aligned}
& P[G(t)]=\text { const, } \\
& Q[\Phi(t)]=\text { const. }
\end{aligned}
$$

The creep and relaxation functions constitute a transformation pair. If we designate the respective Laplace transforms by $\hat{\Phi}(s)$ and $\hat{G}(s)$, the following identity is valid:

$$
s^{2} \hat{\Phi}(s) \hat{G}(s) \equiv 1 .
$$




\subsubsection{General Solution Procedure by Lee and Radok (1960)}

The material behavior of viscoelastic materials is time-dependent. The state of a viscoelastic contact is, therefore, dependent on its loading history. In the case of the normal contact problem, the sole relationship that is independent of the loading history is the contact configuration, i.e., the relationship

$$
d=g(a)
$$

between the indentation depth $d$ and the contact radius $a$. Even this is valid only when the contact radius monotonically increases over time. In this case, the relationship is defined entirely by the form of the indenter but is independent of rheology. On the basis of this idea and the form of the fundamental field equations of elasticity and viscoelasticity theory, Lee (1955) proposed a method to derive the viscoelastic solution from the solution of the elastic problem. This method, based on the Laplace transform, was generalized by Radok (1957). In a joint effort, Lee and Radok (1960) found the solution for the contact of a parabolic indenter, provided that the contact radius is monotonically increasing over time. Hunter (1960) extended this to cases where the contact radius $a$ exhibits a single maximum, facilitating the consideration of, for example, the Hertzian impact problem with a viscoelastic half-space. The solutions for arbitrary loading histories stem from Ting $(1966,1968)$ and Graham $(1965,1967)$. However, it should be noted that the calculation increases in complexity for every additional extremum of the contact radius.

Normal contacts with elastomers frequently appear in testing procedures to determine the material properties of the viscoelastic material. The most frequently used ones are the Shore hardness test (see Sect. 8.3.2), the rebound-indentation test (see Sect. 8.3.3.1), and the impact test (see Sect. 8.3.3.2).

\subsubsection{Contact Radius Increasing Monotonically Over Time}

The solution by Lee and Radok (1960) can be generalized without great difficulty to any rotationally symmetric indenter with the profile $\tilde{z}:=f(r)$. It merely requires the solution of the corresponding elastic problem, which is detailed in Chap. 2 and summarized in the form:

$$
\begin{aligned}
d^{\mathrm{el}} & =d^{\mathrm{el}}(a), \\
F_{N}^{\mathrm{el}} & =F_{N}^{\mathrm{el}}(a), \\
\sigma_{z z}^{\mathrm{el}} & =\sigma_{z z}^{\mathrm{el}}(r ; a)
\end{aligned}
$$

Here, $r$ denotes the radial coordinate, $d$ the indentation depth, $a$ the contact radius, $F_{N}$ the normal force, and $\sigma_{z z}$ the normal stress at the surface of the half-space. The superscript "el" indicates the elastic solution. As previously mentioned, for a monotonically increasing contact radius, the relationship $d=d(a)$ is independent of the material law of the half-space. Using the method by Lee and Radok yields 
the following solution to the viscoelastic problem:

$$
\begin{aligned}
d(t) & =d(a(t)), \\
P\left[\sigma_{z z}(r, t)\right] & =\frac{1}{2 G_{0}} Q\left[\sigma_{z z}^{\mathrm{el}}(r ; a(t))\right], \\
P\left[F_{N}(t)\right] & =\frac{1}{2 G_{0}} Q\left[F_{N}^{\mathrm{el}}(a(t))\right],
\end{aligned}
$$

with the operators $P$ and $Q$ introduced in (8.47) and (8.48). $G_{0}$ is the shear modulus assumed for the elastic solution. Since all elastic stresses are linear in $G_{0}$, the specific value of $G_{0}$ is irrelevant. The solution can also be expressed using the relaxation function:

$$
\begin{gathered}
\sigma_{z z}(r, t)=\frac{1}{G_{0}} \int_{-\infty}^{t} G(t-\tau) \frac{\partial}{\partial \tau}\left\{\sigma_{z z}^{\mathrm{el}}[r ; a(\tau)]\right\} \mathrm{d} \tau, \\
F_{N}(t)=\frac{1}{G_{0}} \int_{-\infty}^{t} G(t-\tau) \frac{\mathrm{d}}{\mathrm{d} \tau}\left\{F_{N}^{\mathrm{el}}[a(\tau)]\right\} \mathrm{d} \tau .
\end{gathered}
$$

The stress vanishes outside the contact, i.e., for $r>a(t)$. This must be taken into account for the integrations. Two particularly elegant and simple cases occur when either the contact radius or the entire normal force is kept constant (see Sects. 8.3.1 and 8.3.2).

\subsection{Explicit Solutions for Contacts with Viscoelastic Media Using the MDR}

\subsubsection{Indentation of a Cylindrical Punch in a Linear Viscous Fluid}

In this section, we will consider the indentation of a rigid cylindrical punch of radius $a$ with the constant force $F_{N}$ in a linear viscous half-space (viscosity $\eta$, no gravity, no capillarity), as demonstrated in Fig. 8.9. We will calculate the indentation velocity and the indentation depth as a function of time.

Fig. 8.9 Indentation of a cylindrical punch in a viscous half-space

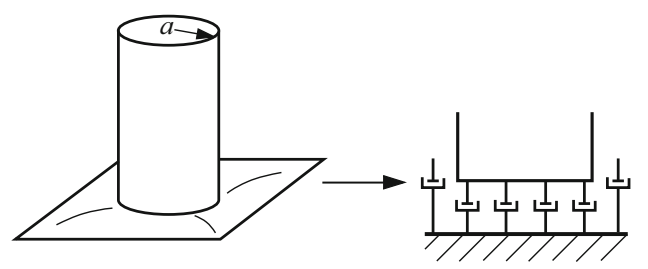


The first step is to determine the equivalent one-dimensional profile using (8.44):

$$
g(x)= \begin{cases}0, & |x|<a, \\ \infty, & |x| \geq a .\end{cases}
$$

The second step is to define the Winkler foundation in accordance with (8.42). For the "spring" force, we obtained $\Delta F_{N}=4 \eta \dot{w}_{1 D} \Delta x=4 \eta \dot{d}(t) \Delta x$. At a contact radius $a(t)$, the vertical force is equal to the individual spring force multiplied by $2 a / \Delta x$, which is the number of springs in the contact:

$$
F_{N}=8 \eta a(t) \dot{d}(t)
$$

In the case of a cylindrical punch, the contact radius remains constant and equal to a. The force is given by:

$$
F_{N}=8 \eta a \dot{d} .
$$

If the force is constant, integration of (8.58) with the initial condition $d(0)=0$ yields:

$$
F_{N} t=8 \eta \operatorname{ad}(t) .
$$

The indentation depth as a function of time is then given by:

$$
d(t)=\frac{F_{N} t}{8 \eta a}
$$

\subsubsection{Indentation of a Cone in a Linear Viscous Fluid}

In this section, we will consider the indentation of a rigid cone $f(r)=\tan \theta \cdot|r|$ with the constant force $F_{N}$ into a linear viscous half-space (viscosity $\eta$, no gravity, no capillarity), as demonstrated in Fig. 8.10. We will calculate the indentation speed and indentation depth as a function of time.

First the equivalent one-dimensional profile is determined using (8.44):

$$
g(x)=\frac{\pi}{2}|x| \tan \theta \text {. }
$$

The second step is to define the Winkler foundation in accordance with (8.42). For the "spring" forces we get $\Delta F_{N}=4 \eta \dot{w}_{1 D} \Delta x=4 \eta \dot{d}(t) \Delta x$. The total normal force is determined by (8.57). The relationship between the current contact radius

Fig. 8.10 Indentation of a cone into a viscous half-space

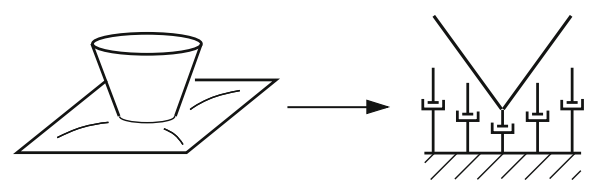


and the indentation depth does not depend on the rheology and follows from the equation $d(t)=g(a(t))$ :

$$
a(t)=\frac{2}{\pi} \frac{d(t)}{\tan \theta} .
$$

Substituting this equation into (8.57) for the force yields:

$$
F_{N}=\frac{16}{\pi \tan \theta} \eta d(t) \dot{d}(t)
$$

If the force is constant, the integration with the initial condition $d(0)=0$ results in:

$$
F_{N} t=\frac{8}{\pi \tan \theta} \eta d(t)^{2}
$$

The indentation depth as a function of time is then given by:

$$
d(t)=\left(\frac{\pi \tan \theta \cdot F_{N} t}{8 \eta}\right)^{1 / 2} .
$$

\subsubsection{Indentation of a Parabolic Indenter into a Linear Viscous Fluid}

In this section, we will look at the indentation of a rigid paraboloid of rotation $f(r)=r^{2} /(2 R)$ (see Fig. 8.11) with the constant force $F_{N}$ in a linear viscous half-space (viscosity $\eta$, no gravity, no capillarity). We will calculate the indentation speed and indentation depth as a function of time.

First the equivalent one-dimensional profile is determined using (8.44):

$$
g(x)=x^{2} / R
$$

For the second step, we will define the Winkler foundation in accordance with (8.42). For the "spring" forces we get $\Delta F_{N}=4 \eta \dot{w}_{1 D} \Delta x=4 \eta \dot{d}(t) \Delta x$. The total normal force is determined by (8.57). The relationship between the current contact radius and the indentation depth does not depend on the rheology and follows from the equation $d(t)=g(a(t))$ :

$$
a(t)=\sqrt{R d(t)} .
$$

Substituting the contact radius into the equation for the force yields:

$$
F_{N}=8 \eta R^{1 / 2} \sqrt{d(t)} \dot{d}(t) .
$$

Fig. 8.11 Indentation of a paraboloid in a viscous half-space

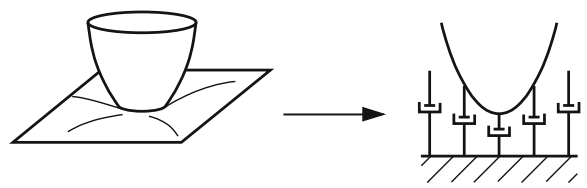


If the force is constant, integration with the initial condition $d(0)=0$ yields:

$$
F_{N} t=\frac{16}{3} \eta R^{1 / 2} d(t)^{3 / 2} .
$$

The indentation depth as a function of time is then given by:

$$
d(t)=\left(\frac{3 F_{N} t}{16 \eta R^{1 / 2}}\right)^{2 / 3} .
$$

\subsubsection{Indentation of a Cone in a Kelvin Medium}

Let us now consider the indentation of a cone in a Kelvin medium with shear modulus $G$ and dynamic viscosity $\eta$. In the first step, the equivalent one-dimensional profile is determined by (8.44): $g(x)=\tan \theta \cdot|x| \cdot \pi / 2$. The contact radius is determined by the equation $d=g(a)$, thus $a=(2 / \pi)(d / \tan \theta)$. We now have to use a superposition of the elastic contribution for the force (see Sect. 2.5.2 in Chap. 2):

$$
F_{N, \mathrm{el}}=\frac{8 G}{\pi} \frac{d^{2}}{\tan \theta}
$$

and the viscous contribution (see (8.63)):

$$
F_{N}=\frac{8 G}{\pi} \frac{d^{2}}{\tan \theta}+\frac{16 \eta}{\pi \tan \theta} d \dot{d} .
$$

This equation can be written in the form

$$
\frac{\pi \tan \theta \cdot F_{N}}{8 G}=d^{2}+2 \tau d \dot{d}=d^{2}+\tau \frac{\mathrm{d}\left(d^{2}\right)}{\mathrm{d} t},
$$

where $\tau=\eta / G$ is the relaxation time of the medium. Integration of this equation with the initial condition $d(0)=0$ yields:

$$
d^{2}(t)=\frac{\pi \tan \theta \cdot F_{N}}{8 G}\left(1-e^{-t / \tau}\right) .
$$

\subsubsection{Indentation of a Rigid Cylindrical Indenter into a "Standard Medium"}

We will now look at the indentation of a rigid cylindrical indenter with radius $a$ into an elastomer, which will be described using the "standard model" (see Fig. 8.6).

The standard model of an elastomer consists of a Maxwell element (seriesconnected stiffness $G_{2}$ and damping $\eta$ ) and a parallel stiffness $G_{1}$. The onedimensional counterpart is a foundation of elements at a distance $\Delta x$, whose 
individual components are characterized by the parameters $4 G_{1} \Delta x, 4 G_{2} \Delta x$, and $4 \eta \Delta x$. The equivalent one-dimensional indenter is a rectangle whose side has the length $2 a$. For the normal force the following applies:

$$
F_{N}=8 G_{1} a d+8 G_{2} a\left(d-w_{1,1 D}\right),
$$

where $w_{1,1 D}$ satisfies the following equation:

$$
d=w_{1,1 D}+\tau \dot{w}_{1,1 D},
$$

with $\tau=\eta / G_{2}$. The solution of these equations with the initial conditions $d(0)=0$ and $w_{1,1 D}(0)=0$ yields to:

$$
\begin{aligned}
w_{1,1 D}(t) & =\frac{F_{N}}{8 G_{1} a}\left(1-\exp \left(-\frac{G_{1} t}{\tau\left(G_{1}+G_{2}\right)}\right)\right), \\
d(t) & =\frac{F_{N}}{8 G_{1} a}\left(1-\frac{G_{2}}{G_{1}+G_{2}} \exp \left(-\frac{G_{1} t}{\tau\left(G_{1}+G_{2}\right)}\right)\right) .
\end{aligned}
$$

In the limiting case $G_{2} \gg G_{1}$ we recover the result for a Kelvin body:

$$
d(t)=\frac{F_{N}}{8 G_{1} a}\left(1-\exp \left(-\frac{G_{1} t}{\eta}\right)\right) .
$$

\subsection{Explicit Solutions for Contacts with Viscoelastic Media by Lee and Radok (1960)}

\subsubsection{Constant Contact Radius}

For a constant contact radius, i.e., the limiting case of the requisite monotonic behavior, the right-hand side of (8.54) is populated by time-invariant terms. The comparison to (8.50) immediately yields:

$$
\begin{aligned}
\sigma_{z z}(r, t) & =\frac{1}{G(t=0)} \sigma_{z z}^{\mathrm{el}}(r ; a) G(t), \\
F_{N}(t) & =\frac{1}{G(t=0)} F_{N}^{\mathrm{el}}(a) G(t),
\end{aligned}
$$

with the relaxation function $G(t)$.

\subsubsection{Constant Normal Force (Shore Hardness Test, DIN EN ISO 868)}

According to (8.54), for a constant normal force $F_{N}(t)=$ const $=F_{0}$, it is valid that $P\left[F_{N}(t)\right]=$ const. The comparison to (8.50) yields the relationship:

$$
F_{N}^{\mathrm{el}}(a(t))=F_{0} \Phi(t),
$$


with $\Phi(t)$ denoting the (normalized) creep function. A few examples for (8.80) were explicitly considered in Sect. 8.2.

From (8.80) we can design a very simple measurement procedure of the creep function: an indenter can be placed on the viscoelastic half-space and the indentation depth resulting from its own weight can be measured as a function of time.

Another important application of (8.80) is measuring the hardness of viscoelastic media according to Shore (DIN EN ISO 868). It involves indenting a test piece with a rigid indenter by applying a constant force $F_{0}$ for a predetermined amount of time and measuring the achieved indentation depth at the end of the test. This indentation depth is then the determining measure for the Shore hardness. The values of the normal force and indentation time, as well as the particular indenter geometry vary according to the specific test form (Shore-A, Shore-D, etc.). If the creep function of the material to be inspected is known, the result of the hardness test can be predicted by (8.80) (within the scope of the fundamental assumptions of the equation, i.e., linear material behavior and validity of the half-space hypothesis). As indenting bodies: a cone for the Shore-D test (see Sect. 8.3.2.1) and a truncated cone for Shore-A test (see Sect. 8.3.2.2) are utilized. We will also consider the indenter profile in the form of a power-law (see Sect. 8.3.2.3) as, through a Taylor series expansion, it can function as a building block of a generalized Shore test for any differentiable indenter profile.

\subsubsection{Shore-D: The Cone in Contact with a Standard Solid}

For illustration purposes, we will restrict the consideration to the viscoelastic material model of the standard solid, for which we determined the normalized creep function in Table 8.1 (Sect. 8.1.3.4) to be:

$$
\Phi(t)=\frac{1}{k}\left[1-(1-k) \exp \left(-\frac{k t}{\tau}\right)\right] .
$$

Here, $k$ is the ratio between the static modulus and glass modulus, while $\tau$ is the characteristic relaxation time of the standard solid model.

In Chap. 2 of this book (see Sect. 2.5.2) it was demonstrated that, for the conical indenter with the slope angle $\theta$, which was first considered in viscoelastic contact by Graham (1965), the normal force in the elastic case is proportional to the square of the indentation depth $d$ :

$$
F_{N}^{\mathrm{el}} \sim d^{2} .
$$

Equation (8.80) and the creep function (8.81) of the standard body give the following relationship for the indentation depth as a function of time:

$$
\frac{d(t)}{d(t=0)}=\sqrt{\frac{1}{k}\left[1-(1-k) \exp \left(-\frac{k t}{\tau}\right)\right]} .
$$


Fig. 8.12 Dependency of the normalized indentation depth on the normalized time in logarithmic representation for the Shore hardness test of a standard solid medium with a conical indenter

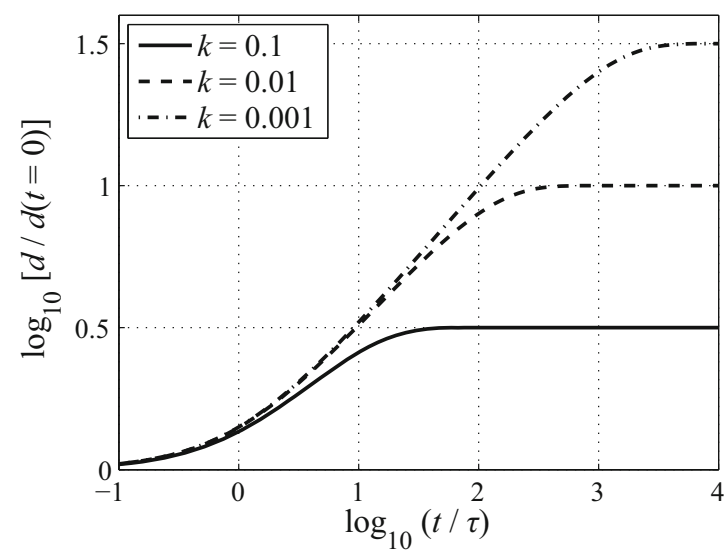

The indentation depth at the beginning of the test $d(t=0)$ is then the instantaneous elastic solution with the glass modulus $G_{0}$ (see Sect. 2.5.2):

$$
d(t=0)=\sqrt{\frac{\pi F \tan \theta_{0}}{8 G_{0}}} .
$$

Solution (8.83) is shown in normalized logarithmic representation in Fig. 8.12.

\subsubsection{Shore-A: The Truncated Cone in Contact with a Standard Solid}

The Shore A test, which is used for softer materials, is carried out with a truncated cone as the indenting body. This body has the rotationally symmetric profile

$$
f(r)= \begin{cases}0, & r \leq b, \\ (r-b) \tan \theta, & r>b\end{cases}
$$

with the radius $b$ at the blunt end and the conical inclination angle $\theta$ (note that the complementary angle $\theta^{*}=\pi / 2-\theta$ is used in the Shore hardness measurement standard). The elastic solution needed to apply (8.80) for this contact problem was derived in Chap. 2 (Sect. 2.5.9) and is described by:

$$
\begin{aligned}
d(a) & =a \tan \theta \arccos \left(\frac{b}{a}\right), \\
F_{N}^{\mathrm{el}}(a) & =4 G \tan \theta a^{2}\left[\arccos \left(\frac{b}{a}\right)+\frac{b}{a} \sqrt{1-\frac{b^{2}}{a^{2}}}\right] .
\end{aligned}
$$


The contact radius $a$ as a function of time during the hardness measurement of a standard medium, therefore, results implicitly by solving the equation

$$
\begin{aligned}
& \frac{G_{0} \tan \theta a^{2}}{F_{0}}\left[\arccos \left(\frac{b}{a}\right)+\frac{b}{a} \sqrt{1-\frac{b^{2}}{a^{2}}}\right] \\
& =\frac{1}{k}\left[1-(1-k) \exp \left(-\frac{k t}{\tau}\right)\right] .
\end{aligned}
$$

From the contact radius the remaining indentation depth, and thus the Shore hardness, can then be determined with the first of the two equations given in (8.86). For $b=0$, the results for the fully conical indenter are reproduced.

\subsubsection{Generalized Shore Test with an Indenter in the Form of a Power-Law in Contact with a Standard Body}

For an indenter with the profile

$$
f(r)=c r^{n}, \quad n \in \mathbb{R}^{+},
$$

where $n$ is a positive real number and $c$ a constant coefficient, the relationship between the normal force and the indentation depth in the elastic case has been shown in Chap. 2 of this book (Sect. 2.5.8):

$$
F_{N}^{\mathrm{el}} \sim d^{\frac{n+1}{n}} .
$$

With (8.80) and the creep function (8.81) of a standard medium, we get the following indentation depth as a function of time:

$$
\frac{d(t)}{d(t=0)}=\left\{\frac{1}{k}\left[1-(1-k) \exp \left(-\frac{k t}{\tau}\right)\right]\right\}^{\frac{n}{n+1}} .
$$

Then the instantaneous indentation depth $d(t=0)$ results from the elastic solution with the glass module $G_{0}$ :

$$
d(t=0)=\left\{\frac{F_{0}[\kappa(n) c]^{n}}{8 G} \frac{n+1}{n}\right\}^{\frac{n}{n+1}},
$$

with the stretch factor, which depends on the exponent of the profile

$$
\kappa(n):=\sqrt{\pi} \frac{\Gamma(n / 2+1)}{\Gamma[(n+1) / 2]},
$$

where we have made use of the gamma function

$$
\Gamma(z):=\int_{0}^{\infty} t^{z-1} \exp (-t) \mathrm{d} t .
$$

For $n=1$ the results of the conical indenter demonstrated here can be reproduced. 


\subsubsection{Non-Monotonic Indentation: Contact Radius with a Single Maximum}

In this section, we consider loading histories consisting of an indentation and rebound phase. Let the contact radius have its maximum value at the time of reversal $t_{m}$ and let the time $t=0$ be zero. The latter condition presents a restriction to the generality, but it is surely the most common case. A possible graph of the contact radius is depicted in Fig. 8.13.

The first step is to solve the contact problem for the indentation phase $t \leq t_{m}$. We have at our disposal the results of the previous sections and, specifically, we have (8.54). Additionally, there exists an explicit function $t_{1}\left(t^{\prime}\right)$ with $t_{1}<t_{m}$ and $t^{\prime}>t_{m}$ so that (see the Fig. 8.13)

$$
a\left(t_{1}\right)=a\left(t^{\prime}\right) .
$$

For the indentation depth $d(t)$ and the normal force $F_{N}(t)$ during the rebound phase $t>t_{m}$, we obtain the expressions for arbitrary rotationally symmetric indenters determined by Ting (1966):

$$
\begin{aligned}
d(t) & =d^{\mathrm{el}}(a(t))-\int_{t_{m}}^{t} \Phi\left(t-t^{\prime}\right) \frac{\mathrm{d}}{\mathrm{d} t^{\prime}}\left[\int_{t_{1}\left(t^{\prime}\right)}^{t^{\prime}} G\left(t^{\prime}-t^{\prime \prime}\right) \frac{\mathrm{d}}{\mathrm{d} t^{\prime \prime}} d^{\mathrm{el}}\left(a\left(t^{\prime \prime}\right)\right) \mathrm{d} t^{\prime \prime}\right] \mathrm{d} t^{\prime}, \\
F_{N}(t) & =\int_{0}^{t_{1}(t)} G\left(t-t^{\prime}\right) \frac{\mathrm{d}}{\mathrm{d} t^{\prime}} F_{N}^{\mathrm{el}}\left(a\left(t^{\prime}\right)\right) \mathrm{d} t^{\prime},
\end{aligned}
$$

with the normalized creep function $\Phi$, the normalized relaxation function $G$, and the elastic solutions indicated by the superscript "el", which is discussed in Chap. 2.

In the case of the parabolic indenter, the results from (8.95) were already known to Hunter (1960). Furthermore, Ting (1968) and Graham (1967) found the solutions for arbitrary counts of maxima and minima of the contact radius. Regrettably, the analytical treatment of these increasingly interlinked differentiations and integrations is not practically viable.

Of course one can conceive various loading protocols where the contact radius has a single maximum and the contact problem thus decomposes into an indentation and rebound phase. Two technically relevant cases are discussed in the literature:

Fig. 8.13 Schematic graph of the contact radius

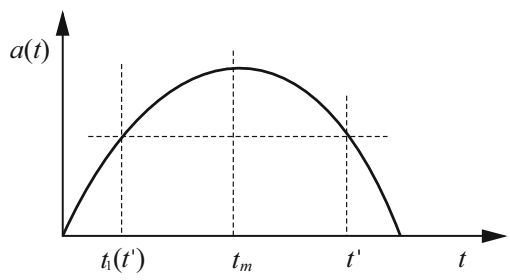


rebound-indentation testing in which the first phase is displacement-controlled and the force is zero in the second phase, and the linear centric impact problem, which of equal importance for various testing procedures.

\subsubsection{Material Tests Using the Rebound-Indentation Procedure}

In rebound-indentation testing, an indenter is pressed under displacement-control into a viscoelastic half-space, e.g., with a constant velocity $v_{0}$ :

$$
d^{I}(t)=v_{0} t .
$$

The superscript " $I$ " indicates the indentation phase $t \leq t_{m}$. The indenter is subsequently released and the half-space relaxes. By measuring the indentation depth during relaxation, it is possible to determine the time-dependent material functions of the elastomer. The protocols of the indentations are displayed in Figs. 8.14 and 8.15 .

Argatov and Popov (2016) were able to prove that the indentation depth during the rebound phase fulfills the relationship

$$
d^{I I}(t)=\int_{0}^{t_{m}} \mathrm{~K}\left(t-t^{\prime}, t_{m}-t^{\prime}\right) \frac{\mathrm{d}}{\mathrm{d} t^{\prime}} d^{I}\left(t^{\prime}\right) \mathrm{d} t^{\prime}=v_{0} \int_{0}^{t_{m}} K\left(t-t^{\prime}, t_{m}-t^{\prime}\right) \mathrm{d} t^{\prime},
$$

independent of the actual form of the indenter Equation (8.97). for the flat cylindrical punch was already derived by Argatov and Mishuris (2011) and for the parabolic indenter by Argatov (2012). The function $K\left(t, t^{\prime}\right)$, which was previously utilized by Greenwood (2010) for an alternative presentation of the solutions by Ting (1966) and which presents the stress response via a unit deformation applied at $t=0$ and

Fig. 8.14 Protocol of the indentation depth during rebound-indentation testing

Fig. 8.15 Protocol of the normal force during reboundindentation testing. The rebound phase occurs under zero force
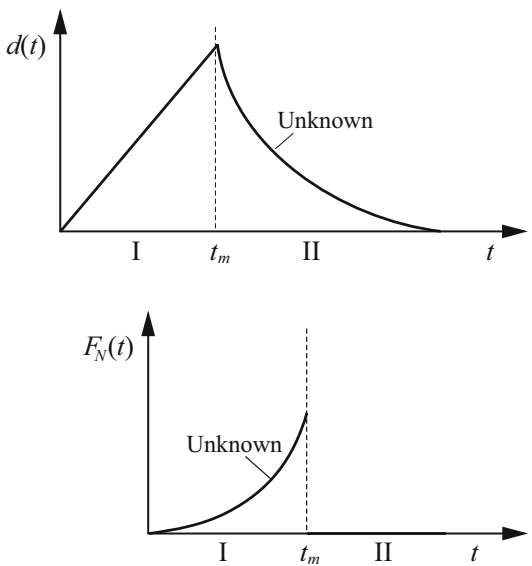
Fig. 8.16 The function $K\left(t, t^{\prime}\right)$ from (8.99) for the three-element standard solid with $k=0.1$

Fig. 8.17 The function $K\left(t, t^{\prime}\right)$ for the three-element standard solid with $k=0.5$
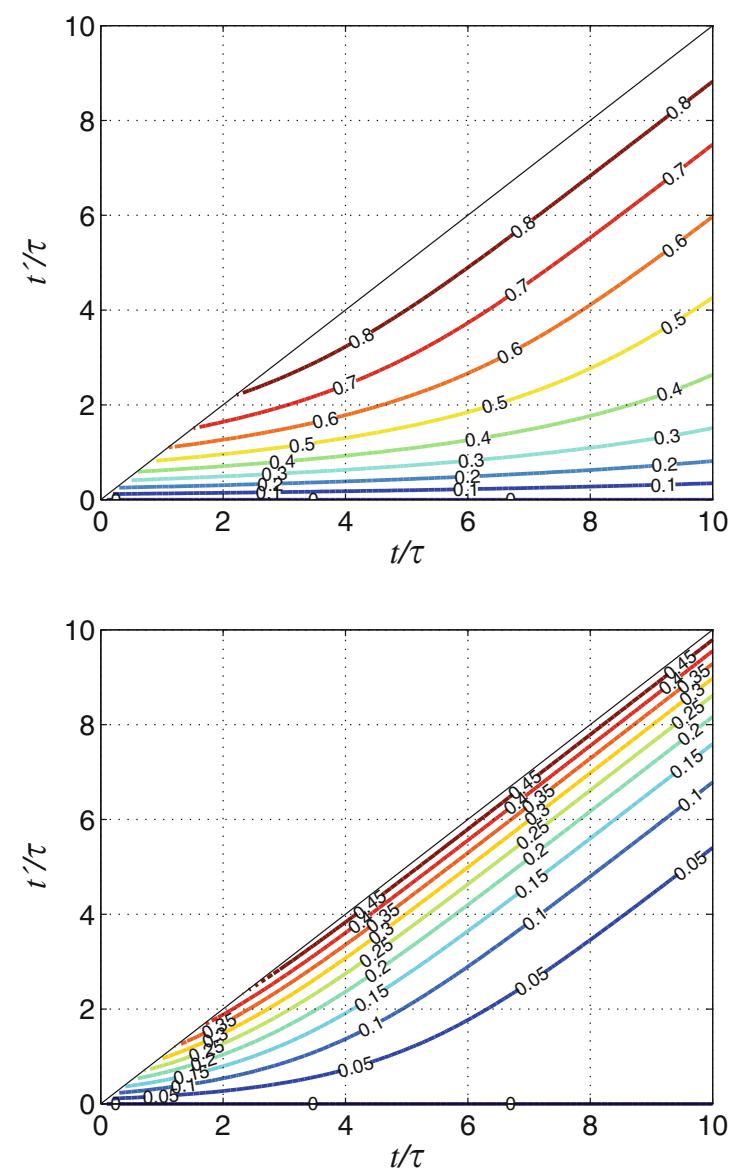

removed at $t=t^{\prime}$, is connected to functions $\Phi$ and $\Psi$ by the relationship:

$$
K\left(t, t^{\prime}\right)=1-\Psi(t)+\int_{t^{\prime}}^{t} \Psi\left(t^{\prime \prime}\right) \frac{\partial}{\partial t^{\prime \prime}} \Phi\left(t-t^{\prime \prime}\right) \mathrm{d} t^{\prime \prime}, \quad t>t^{\prime}
$$

For the three-element standard solid with $k=G_{\infty} / G_{0}$ and the characteristic relaxation time $\tau$, the expression is:

$$
K\left(t, t^{\prime}\right)=(1-k)\left(1-\exp \left(-\frac{t^{\prime}}{\tau}\right)\right) \exp \left(-\frac{k\left(t-t^{\prime}\right)}{\tau}\right), \quad t>t^{\prime} .
$$

A normalized representation of this function for two different values of $k$ is given in Figs. 8.16 and 8.17. 


\subsubsection{The Hertzian Impact Problem for a Viscoelastic Half-Space}

Another simple test method for determining the viscoelastic properties of elastomers is rebound testing, which is usually carried out with spherical impact bodies. From the measured number of impacts during the experiment, conclusions about the dynamic material behavior of the rubber can be drawn.

Hunter (1960) looked at the Hertzian impact problem, i.e., the straight, centric, frictionless impact of a rigid sphere, with a viscoelastic half-space when the peak time is small compared to the smallest characteristic relaxation time $\tau$ of the elastomer. In this case, the creep and relaxation functions of the elastomer in normalized representation can be approximated by the expressions of the Maxwell body:

$$
\begin{aligned}
\Phi(t) & =1+\frac{t}{\tau}, \\
G(t) & =\exp \left(-\frac{t}{\tau}\right) .
\end{aligned}
$$

The sphere has the radius $R$ and the initial velocity $v_{0}$. The half-space has the glass module $G_{0}$ and the (constant in time) Poisson number $v$. In this case, Hunter determined the following equations of motion for the variable $Z(t)=a^{2}(t) / R$ :

$$
\begin{aligned}
& \ddot{Z}+\frac{1}{\tau}\left(\dot{Z}-v_{0}\right)+C Z^{3 / 2}=0, t \leq t_{m}, \\
& \ddot{Z}-\frac{1}{\tau}\left(3 \dot{Z}+v_{0}\right)+C Z^{3 / 2}=0, \quad t>t_{m},
\end{aligned}
$$

with

$$
C=\frac{8 G_{0} R^{1 / 2}}{3 M(1-v)} .
$$

During the penetration phase, $Z$ corresponds with the indentation depth (which is the position of the sphere) and the equation of motion is exact. The presented equation of motion during the retraction phase, on the other hand, is an approximation that is accurate only for large values of $\tau$ compared to the impact time. Hunter then determined the following approximate solutions for the coefficient of restitution $e$ and the impact duration $T$ :

$$
\begin{aligned}
e & =1-\frac{4}{9} \frac{T_{0}}{\tau}, \\
T & =T_{0}\left(1-0.037 \frac{T_{0}}{\tau}\right) .
\end{aligned}
$$

Here, $T_{0}$ denotes the duration of impact for the purely elastic case, according to Hertz (1882). The viscoelastic impact problem was also examined in detail by Argatov (2013) using an asymptotic approximation. 


\subsection{Normal Contact of Compressible Viscoelastic Media}

Most elastomers can, to a good approximation, be considered incompressible. This was one of the general assumptions in the previous sections of this chapter, with only the shear modulus (complex modulus, relaxation function, creep function, etc.) being the subject of rheological study. Yet there is no theoretical justification requiring this assumption of incompressibility. Indeed, elastomers generally exhibit a deformation response to hydrostatic compression, and a relaxation function and creep function associated with this volume deformation.

In the following sections, we will outline some possibilities on how to take the compressibility of the viscoelastic medium into account for contact problems. At several stages we will refer back to various concepts introduced earlier in this chapter.

\subsubsection{The Compressible Viscoelastic Material Law}

Equation (8.47) in Sect. 8.1.5 introduced the general material law of a linear viscoelastic medium for the traceless components (belonging to the shear components) of the stress and deformation tensor:

$$
P_{S}\left[s_{i j}(t)\right]=Q_{S}\left[e_{i j}(t)\right] .
$$

Here, $s_{i j}$ denotes the traceless component of the stress tensor $\sigma_{i j}$ and $e_{i j}$ denotes the traceless component of the deformation tensor $\varepsilon_{i j} . P$ and $Q$ are linear operators of the form

$$
\begin{aligned}
P_{S} & :=\sum_{k=0}^{\infty} p_{S, k} \frac{\mathrm{d}^{k}}{\mathrm{~d} t^{k}}, \\
Q_{S} & :=\sum_{k=0}^{\infty} q_{S, k} \frac{\mathrm{d}^{k}}{\mathrm{~d} t^{k}},
\end{aligned}
$$

with an added index " $S$ " to emphasize that we are dealing with the shear component of the deformation. For the volume components, i.e., the trace components, an analogous material law for the most general case can be formulated:

$$
P_{V}\left[\sigma_{i i}(t)\right]=Q_{V}\left[\varepsilon_{i i}(t)\right]
$$

with analogous operators:

$$
\begin{aligned}
P_{V} & :=\sum_{k=0}^{\infty} p_{V, k} \frac{\mathrm{d}^{k}}{\mathrm{~d} t^{k}}, \\
Q_{V} & :=\sum_{k=0}^{\infty} q_{V, k} \frac{\mathrm{d}^{k}}{\mathrm{~d} t^{k}} .
\end{aligned}
$$


For example, let us consider a compressible Kelvin medium for which the stress state has only a purely linear-elastic and a purely linear viscous component. In this case, the material law that we already split into its shear and volume components is:

$$
\begin{aligned}
\sigma_{l l} & =3 K \varepsilon_{l l}+3 \xi \dot{\varepsilon}_{l l}, \\
s_{i j} & =2 G e_{i j}+2 \eta \dot{e}_{i j},
\end{aligned}
$$

which means the previously introduced differential operators have the form:

$$
\begin{gathered}
P_{S}=P_{V}=1 \\
Q_{V}=3 K+3 \xi \frac{\partial}{\partial t}, \\
Q_{S}=2 G+2 \eta \frac{\partial}{\partial t} .
\end{gathered}
$$

Here, $G$ and $K$ are the shear and bulk modulus, while $\eta$ and $\xi$ are the dynamic shear and volume viscosity of the medium, respectively.

\subsubsection{Is MDR Mapping of the Compressible Normal Contact Problem Possible?}

The solution process of the normal contact problem begins, once again, with the fundamental solution for the displacement $w$ of a half-space under the effect of a normal point force $F_{N}$, which acts on the origin starting at time $t=0$ and is then held constant. In the elastic case, the fundamental solution to the normal displacement of the surface is the well-known solution by Boussinesq given in (2.2),

$$
w^{e l}(r, t)=\frac{F_{N} H(t)}{\pi E^{*} r}=\frac{F_{N} H(t)}{4 \pi G r} \frac{3 K+4 G}{3 K+G},
$$

with the effective elasticity modulus $E^{*}$. According to the principle of Radok (1957), solving the viscoelastic problem involves the Laplace transform of the elastic solution (owed to the analogous forms of the elastic and viscoelastic field equations) with the accompanying substitution of the elastic moduli through the operators $P$ and $Q$. The following equation is obtained for the Laplace transform (all transformed quantities are indicated by a "hat") of the desired fundamental solution:

$$
\hat{w}(r, s)=\frac{F_{N}}{2 \pi r s} \frac{\hat{Q}_{V}+2 \hat{Q}_{S}}{\hat{Q}_{S}\left[\hat{Q}_{V}+\hat{Q}_{S} / 2\right]} .
$$

However, the transforms of the operators are polynomials in $s$,

$$
\begin{aligned}
& \hat{Q}_{S}=\sum_{k=0}^{\infty} q_{S, k} s^{k}, \\
& \hat{Q}_{V}=\sum_{k=0}^{\infty} q_{V, k} s^{k} .
\end{aligned}
$$


In other words, (8.111) can be written in the general form:

$$
\hat{w}(r, s)=\frac{F_{N}}{4 \pi r} \hat{\Phi}(s),
$$

with

$$
\hat{\Phi}(s):=\frac{2}{s} \frac{\sum_{k=0}^{\infty} q_{V, k} s^{k}+2 \sum_{k=0}^{\infty} q_{S, k} s^{k}}{\sum_{k=0}^{\infty} q_{S, k} s^{k}\left(\sum_{k=0}^{\infty} q_{V, k} s^{k}+\frac{1}{2} \sum_{k=0}^{\infty} q_{S, k} s^{k}\right)},
$$

which, after an inverse transform into the time domain, leads to the desired solution

$$
w(r, t)=\frac{F_{N}}{4 \pi r} \Phi(t)
$$

Yet this is the fundamental solution for an incompressible medium with the (dimensional, shear) creep function $\Phi(t)$. This means that the normal contact problem for a compressible viscoelastic material can always be reduced to an equivalent problem of an incompressible medium, assuming that the material law of the compressible body in the form (8.104) and (8.106) is known and the inverse Laplace transform of the expression (8.114) exists.

In particular, the relationship (8.51) between the creep function and the timedependent shear modulus $G(t)$,

$$
s^{2} \hat{\Phi}(s) \hat{G}(s)=1,
$$

allows finding the time-dependent shear modulus by using the inverse Laplace transform of the expression

$$
\hat{G}(s)=\frac{\sum_{k=0}^{\infty} q_{S, k} s^{k}\left(\sum_{k=0}^{\infty} q_{V, k} s^{k}+\frac{1}{2} \sum_{k=0}^{\infty} q_{S, k} s^{k}\right)}{2 s\left(\sum_{k=0}^{\infty} q_{V, k} s^{k}+2 \sum_{k=0}^{\infty} q_{S, k} s^{k}\right)} .
$$

Thus all previously derived MDR relations for the normal contact from previous sections can also be used in the case of compressible viscoelastic media.

\subsubsection{Normal Contact of a Compressible Kelvin Element}

To illustrate the relationships derived in the previous section, we will provide a brief demonstration of the transformation of the compressible normal contact to the corresponding incompressible problem for an incompressible Kelvin element with the general material law (8.108).

Inserting the operators (8.109) into (8.114) leads to the following expression for the Laplace transform of the shear creep function of the incompressible medium in question:

$$
\hat{\Phi}(s)=\frac{1}{s} \frac{3 K+4 G+(3 \xi+4 \eta) s}{(G+\eta s)[3 K+G+(3 \xi+\eta) s]} .
$$


The inverse transform into the time domain yields the creep function

$$
\Phi(t)=\frac{1}{G}\left[1-\exp \left(-\frac{G}{\eta} t\right)\right]+\frac{3}{3 K+G}\left[1-\exp \left(-\frac{3 K+G}{3 \xi+\eta} t\right)\right]
$$

and the time-dependent modulus (to avoid any confusion with the shear modulus of the original compressible medium, we add the index "ink")

$$
\begin{aligned}
G_{\text {ink }}(t)= & \eta \frac{3 \xi+\eta}{3 \xi+4 \eta} \delta(t)+G \frac{3 K+G}{3 K+4 G} \\
& +\frac{27(G \xi-K \eta)^{2}}{(3 K+4 G)(3 \xi+4 \eta)^{2}} \exp \left(-\frac{3 K+4 G}{3 \xi+4 \eta} t\right)
\end{aligned}
$$

Here, $\delta(t)$ denotes the Dirac function. In the limiting case of an incompressible medium, we obtain the known solution

$$
\lim _{K \rightarrow \infty} G_{\text {ink }}(t)=G+\eta \delta(t) .
$$

The limiting case of rapid relaxation

$$
\frac{3 \xi+4 \eta}{3 K+4 G} \ll t
$$

also results in an incompressible Kelvin body with

$$
G_{\mathrm{ink}}(t) \approx G \frac{3 K+G}{3 K+4 G}+\left(\eta \frac{3 \xi+\eta}{3 \xi+4 \eta}+\frac{27(G \xi-K \eta)^{2}}{(3 K+4 G)^{2}(3 \xi+4 \eta)}\right) \delta(t) .
$$

It should be noted that the creep function (8.119) and the time-dependent modulus (8.120) can be interpreted in different ways in terms of rheological models (and consequently also for the purposes of the MDR). For example, the modulus (8.120) can be produced by a parallel arrangement of a Kelvin element and a Maxwell element (see Fig. 8.18) with the material parameters of the rheological models set to ( $v$ is the Poisson's ratio of the medium):

$$
\begin{gathered}
G_{1}=G \frac{3 K+G}{3 K+4 G}=\frac{G}{2(1-v)}, \quad G_{2}=\frac{27(G \xi-K \eta)^{2}}{(3 K+4 G)(3 \xi+4 \eta)^{2}}, \\
\eta_{1}=\eta \frac{3 \xi+\eta}{3 \xi+4 \eta}, \quad \eta_{2}=\frac{27(G \xi-K \eta)^{2}}{(3 K+4 G)^{2}(3 \xi+4 \eta)}, \\
\eta_{1}+\eta_{2}=\frac{4 \eta\left(1-v+v^{2}\right)+3 \xi(1-2 v)^{2}}{12(1-v)^{2}} .
\end{gathered}
$$

However, other rheological models are also possible. For instance, the form of the creep function (8.119) suggests the possibility of a serial arrangement of two 
Fig. 8.18 Rheological element to model a compressible Kelvin body within the framework of the MDR

Fig. 8.19 Element for a compressible Kelvin body in the limiting case of rapid relaxation
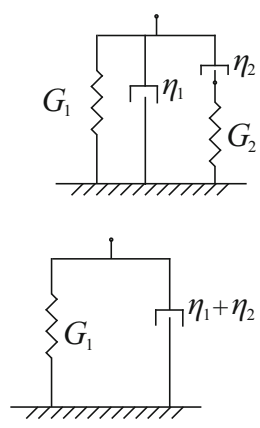

Fig. 8.20 Rheological element for an incompressible Kelvin body

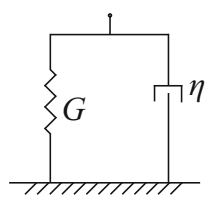

suitably chosen Kelvin elements. The rheological models for the limiting case of fast relaxation and for the incompressible body are shown in Figs. 8.19 and 8.20.

Once the compressible normal contact problem has been reduced to the equivalent incompressible problem and the corresponding rheological model has been identified, all previously derived MDR rules pertaining to the solutions of incompressible problems can be applied. As an example, Brilliantov et al. (2015) derived the dissipating force during the impact of two spheres in the limiting case given by (8.122). using developments from perturbation theory. Based on the aforementioned derivations and the relationships of the incompressible MDR model, it is immediately obvious that, for an arbitrary axially symmetric indenter, this force is given by:

$$
F_{\text {dis }}=8\left(\eta_{1}+\eta_{2}\right) a \dot{d}=\frac{2 a \dot{d}}{(1-v)^{2}}\left[\frac{4}{3} \eta\left(1-v+v^{2}\right)+\xi(1-2 v)^{2}\right],
$$

which (in the case of impacting spheres in the Hertzian approximation) naturally corresponds to the result of Brilliantov et al. In (8.125) $a$, as always, refers to the contact radius and $d$ refers to the indentation depth.

\subsection{Fretting Wear of Elastomers}

Contacts of rigid bodies with polymers or elastomers occur in many applications. If they are moving relative to one another, then the surfaces in such contacts are subjected to wear. Counterintuitively, the endangered partner is not necessarily the softer polymer piece but may well be the metal surface (Higham et al. 1978). Whether there is wear to the more rigid or to the softer contact partner depends on many load and material parameters. In the following, we explore the idea that the 
"rigid" indenter wears while the elastic deformation is solely due to the elasticity of the polymer.

Let us consider an axially symmetric rigid profile which is brought into contact with a polymer (average indentation depth $d_{0}$ ) and is subjected to vibrations of the amplitude $\Delta u^{(0)}$ in the tangential direction and vibrations of the amplitude $\Delta w^{(0)}$ in the normal direction:

$$
\begin{aligned}
d(t) & =d_{0}+\Delta w^{(0)} \cos (\omega t), \\
u^{(0)}(t) & =\Delta u^{(0)} \cos (\omega t+\varphi) .
\end{aligned}
$$

Under the assumption of complete stick in the entire contact area, the tangential stresses at the edge of the contact would exhibit a singularity as in the elastic case. This means that the no-slip condition cannot be met in the vicinity of the edge of the contact. Thus, even small amplitudes lead to the formation of a ring-shaped slip zone at the edge of the contact which results in wear. The inner zone remains sticking. The existence of this stick zone was demonstrated by Barber et al. (2011) for arbitrary two-dimensional topographies (not necessarily with a singular, connected contact area) under very general assumptions and for three-dimensional topographies under the assumptions of the theory of Cattaneo and Mindlin.

In this book, we will restrict our consideration to axially symmetric contacts. Furthermore, we assume that the friction in the contact obeys the local form of the Coulomb law of friction: the surfaces remain sticking if the tangential stress $\tau$ is lower than the normal pressure $p$ multiplied by the coefficient of friction $\mu$, and that the tangential stress remains constant once slip sets in:

$$
\begin{array}{ll}
|\tau|<\mu p, & \text { stick, } \\
|\tau|=\mu p, & \text { slip. }
\end{array}
$$

We refer to the entirety of all points that fulfill the condition $|\tau|<\mu p$ at every point in time as the "permanent stick zone". Due to wear, the stress in the slip zone will decrease over time and be re-distributed to the stick zone. We can assume that the original stick zone remains sticking over the course of the wear process, while in the area of the original slip zone the slip condition is fulfilled even in the worn state. The progressing wear leads to a continual pressure decrease in the slip zone. This process finally ends when the pressure vanishes completely. In this limit state, the wear rate in the slip zone approaches zero: the system approaches a state in which no further wear occurs. The precise kinetics of this process depend on the form of the wear law. The shape of the profile in its final state, however, does not depend on the details of the wear law and it can be determined in a general form.

We will not presume any particular local wear law. Instead, we will just assume that the following very general conditions are met: (a) wear occurs only where there is a finite relative displacement of the surfaces (the existence of tangential stresses alone is not sufficient for wear); (b) wear occurs only in zones of non-zero pressure. Combined with the assumed Coulomb law of friction, these assumptions alone uniquely determine the limit shape of the worn profile. Indeed, from the 


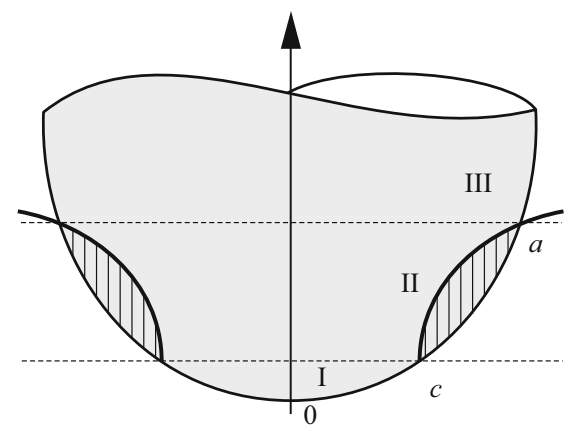

Fig. 8.21 Three zones of the worn profile in the limit state. I is the zone of permanent stick; here the original profile remains unchanged. II is the zone in which the pressure vanishes, even though at maximum indentation the surfaces are in a state of established contact with no pressure. III is the zone in which no contact is established (and consequently no change of the profile occurs)

assumption (a) it follows that the shape of the indenter in the permanent stick zone must match the original (unworn) shape (zone I in Fig. 8.21). Under assumption (b), the pressure outside the permanent stick zone vanishes in the limit state. Yet this unpressurized state should result due to the wear. This means that the surfaces in zone II at the maximum indentation are "just barely touching"; i.e., contact has been established with zero pressure. This in turn means that the worn indenter at the time of maximum indentation must coincide exactly with the shape of the free surface, which would have resulted from the indentation from the original indenter shape within the permanent stick radius (zone II in Fig. 8.21).

According to the half-space hypothesis, small oscillations parallel to the contact surface, independent of their particular oscillation mode (linear oscillations in a single direction, superposition of oscillations in two direction, or torsional oscillations), only result in a tangential displacement of the surfaces that are just barely in contact and therefore cause no wear. Since the shape of a surface that is indented by a non-varying contact area does not depend on the rheology of the medium and matches the one for an elastic body, the shape of the worn indenter in its final state is given by (6.18) which can be found in Chap. 6:

$$
f_{\infty}(r)= \begin{cases}f_{0}(r), & r \leq c, r>a, \\ \frac{2}{\pi}\left[\int_{0}^{c} \frac{g_{0}(x) \mathrm{d} x}{\sqrt{r^{2}-x^{2}}}+d_{\max } \arccos \left(\frac{c}{r}\right)\right], & c<r \leq a .\end{cases}
$$

with

$$
d_{\max }=d_{0}+\Delta w^{(0)}
$$

in which $g_{0}(x)$ is the MDR transformed profile:

$$
g_{0}(x)=|x| \int_{0}^{|x|} \frac{f_{0}^{\prime}(r) \mathrm{d} r}{\sqrt{x^{2}-r^{2}}} .
$$


The solution is uniquely defined by the maximum indentation depth and the radius $c$ of the permanent stick zone. We, therefore, concern ourselves with the calculation of this determining parameter $c$ depending on the loading procedure and the material parameters.

\subsubsection{Determining the Radius $c$ of the Permanent Stick Zone}

In elements of the one-dimensional MDR model, the normal and tangential forces (in the case of an incompressible elastomer) are given by (8.35) and (8.37). The displacements of the "springs" at location $x$ are calculated to:

$$
\begin{aligned}
& w_{1 D}(x, t)=d_{0}-g(x)+\Delta w^{(0)} \cos \omega t, \\
& u_{1 D}(x, t)=\Delta u^{(0)} \cos \left(\omega t+\varphi_{0}\right) .
\end{aligned}
$$

The normal and tangential forces are given by equations

$$
\begin{aligned}
\Delta F_{N}= & 4 \Delta x G^{\prime}(0)\left(d_{0}-g(x)\right) \\
& +4 \Delta x \Delta w^{(0)}\left(G^{\prime}(\omega) \cos \omega t-G^{\prime \prime}(\omega) \sin \omega t\right)
\end{aligned}
$$

and

$$
\Delta F_{x}=\frac{8}{3} \Delta x \Delta u^{(0)}\left(G^{\prime}(\omega) \cos \left(\omega t+\varphi_{0}\right)-G^{\prime \prime}(\omega) \sin \left(\omega t+\varphi_{0}\right)\right),
$$

where $G(\omega)=G^{\prime}(\omega)+i G^{\prime \prime}(\omega)$ is the complex shear modulus; $i$ is the imaginary unit, $G^{\prime}(\omega)$ is the real component of the complex shear modulus (storage modulus), and $G^{\prime \prime}(\omega)$ is the imaginary component of the complex modulus (loss modulus). The no-slip condition reads $\left|\Delta F_{x}\right| \leq \mu \Delta F_{N}$ or

$$
\begin{aligned}
& \frac{2}{3} \Delta u^{(0)}\left|G^{\prime}(\omega) \cos \left(\omega t+\varphi_{0}\right)-G^{\prime \prime}(\omega) \sin \left(\omega t+\varphi_{0}\right)\right| \\
& \leq \mu\left[G^{\prime}(0)\left(d_{0}-g(c)\right)+\Delta w^{(0)}\left(G^{\prime}(\omega) \cos \omega t-G^{\prime \prime}(\omega) \sin \omega t\right)\right] .
\end{aligned}
$$

It follows that:

$$
\begin{aligned}
g(c) \leq d_{0}-\frac{1}{G^{\prime}(0)}[ & \frac{1}{\mu} \frac{2}{3} \Delta u^{(0)}\left|G^{\prime}(\omega) \cos \left(\omega t+\varphi_{0}\right)-G^{\prime \prime}(\omega) \sin \left(\omega t+\varphi_{0}\right)\right| \\
& \left.-\Delta w^{(0)}\left(G^{\prime}(\omega) \cos \omega t-G^{\prime \prime}(\omega) \sin \omega t\right)\right]
\end{aligned}
$$

The radius $c$ of the permanent stick zone is the largest radius, for which there is no slip at any point in time or, alternatively, the smallest of the values where slip is just 
avoided:

$$
\begin{aligned}
g(c)=\min _{t}\left\{d_{0}-\frac{1}{G^{\prime}(0)}[\right. & \frac{1}{\mu} \frac{2}{3} \Delta u^{(0)}\left|G^{\prime}(\omega) \cos \left(\omega t+\varphi_{0}\right)-G^{\prime \prime}(\omega) \sin \left(\omega t+\varphi_{0}\right)\right| \\
& \left.\left.-\Delta w^{(0)}\left(G^{\prime}(\omega) \cos \omega t-G^{\prime \prime}(\omega) \sin \omega t\right)\right]\right\} . \quad(8.136)
\end{aligned}
$$

This equation can also be rewritten in the more compact form, as follows:

$$
g(c)=\min _{t}\left\{d_{0}-\frac{|G(\omega)|}{G^{\prime}(0)}\left[\frac{1}{\mu} \frac{2}{3} \Delta u^{(0)}\left|\cos \left(\Omega+\varphi_{0}\right)\right|-\Delta w^{(0)} \cos \Omega\right]\right\},
$$

with $\Omega=\omega t+\varphi_{1}$, where $\varphi_{1}$ is defined as the phase angle of the complex shear modulus with:

$$
\tan \varphi_{1}=\frac{G^{\prime \prime}(\omega)}{G^{\prime}(\omega)} .
$$

Let us first examine the case in which the phase shift $\varphi_{0}$ between the tangential and normal oscillation is not fixed (e.g., slow "phase creep"). This case would simply require inserting the maximum absolute values of $\left|\cos \left(\Omega+\varphi_{1}\right)\right|$ and $\cos \Omega$ into (8.137):

$$
g(c)=d_{0}-\frac{|G(\omega)|}{G^{\prime}(0)}\left[\frac{1}{\mu} \frac{2}{3}\left|\Delta u^{(0)}\right|+\Delta w^{(0)}\right], \text { (non-fixed phase shift). }
$$

This equation is equally valid for the case of two different incommensurable oscillation frequencies in the normal and the tangential direction.

If the frequencies are equal and the phase shift is fixed by some phase synchronization mechanism, the situation is more complicated. An analysis of (8.137) shows that the smallest stick radius is achieved at $\varphi_{0}=0$. In this case, the radius can also be determined from (8.139). The maximum stick radius is achieved at $\varphi_{0}=\pi / 2$ and is given by the following equation:

$$
g(c)=d_{0}-\frac{|G(\omega)|}{G^{\prime}(0)} \sqrt{\left(\frac{1}{\mu} \frac{2}{3}\left|\Delta u^{(0)}\right|\right)^{2}+\left(\Delta w^{(0)}\right)^{2}} .
$$

The general case of an arbitrary phase shift comprises several special cases, which are presented in research by Mao et al. (2016).

\subsubsection{Fretting Wear of a Parabolic Profile on a Kelvin Body}

As a concrete example for the application of (8.140), we consider a parabolic profile $f_{0}(r)=r^{2} /(2 R)$ in contact with a Kelvin body, which can be represented as a parallel arrangement of an elastic body (shear modulus $G$ ) and a viscous body $\eta$. Accordingly, the elements of the one-dimensional foundation consist of the following elements: 
- In the normal direction, they consist of a spring with the normal stiffness $\Delta k_{z}$ arranged in parallel with a damper with the damping coefficient $\Delta \alpha_{z}$.

- In the horizontal direction, the elements have a tangential stiffness $\Delta k_{x}$ and the damping coefficient $\Delta \alpha_{x}$.

Said values of stiffness and damping coefficients are determined according to (8.38) and (8.39) by the following rules:

$$
\Delta k_{z}=4 G \Delta x, \quad \Delta \alpha_{z}=4 \eta \Delta x, \quad \Delta k_{x}=\frac{8}{3} G \Delta x, \quad \Delta \alpha_{x}=\frac{8}{3} \eta \Delta x .
$$

The MDR profile is, in accordance with (8.130), equal to $g(x)=x^{2} / R$, and the complex shear modulus of the medium is equal to $\hat{G}(\omega)=G+i \omega \eta$. For the rheological quantities $|G(\omega)|$ and $G^{\prime}(0)$ occurring in (8.140) we obtain:

$$
|G(\omega)|=\sqrt{G^{2}+\eta^{2} \omega^{2}} \text { and } \quad G^{\prime}(0)=G .
$$

In the case of $\varphi_{0}=\pi / 2,(8.140)$ takes the form of

$$
\frac{c^{2}}{R}=d_{0}-\sqrt{1+(\omega \tau)^{2}} \sqrt{\left(\frac{1}{\mu} \frac{2}{3}\left|\Delta u^{(0)}\right|\right)^{2}+\left(\Delta w^{(0)}\right)^{2}},
$$

with the introduction of the relaxation time $\tau=\eta / G$.

\section{References}

Argatov, I.I.: An analytical solution of the rebound indentation problem for an isotropic linear viscoelastic layer loaded with a spherical punch. Acta Mech. 223(7), 1441-1453 (2012)

Argatov, I.I.: Mathematical modeling of linear viscoelastic impact: application to drop impact testing of articular cartilage. Tribol. Int. 63, 213-225 (2013)

Argatov, I.I., Mishuris, G.: An analytical solution for a linear viscoelastic layer loaded with a cylindrical punch: evaluation of the rebound indentation test with application for assessing viability of articular cartilage. Mech. Res. Commun. 38(8), 565-568 (2011)

Argatov, I.I., Popov, V.L.: Rebound indentation problem for a viscoelastic half-space and axisymmetric indenter-solution by the method of dimensionality reduction. Z. Angew. Math. Mech. 96(8), 956-967 (2016)

Barber, J.R., Davies, M., Hills, D.A.: Frictional elastic contact with periodic loading. Int. J. Solids Struct. 48(13), 2041-2047 (2011)

Brilliantov, N.V., Pimenova, A.V., Goldobin, D.S.: A dissipative force between colliding viscoelastic bodies: rigorous approach. Europhys. Lett. 109, 14005 (2015)

Graham, G.A.C.: The contact problem in the linear theory of viscoelasticity. Int. J. Eng. Sci. 3(1), 27-46 (1965)

Graham, G.A.C.: The contact problem in the linear theory of viscoelasticity when the timedependent contact area has any number of maxima and minima. Int. J. Eng. Sci. 5(6), 495-514 (1967)

Greenwood, J.A.: Contact between an axi-symmetric indenter and a viscoelastic half-space. Int. J. Mech. Sci. 52(6), 829-835 (2010) 
Hertz, H.: Über die Berührung fester elastischer Körper. J. Reine Angew. Math. 92, 156-171 (1882)

Higham, P.A., Stott, F.H., Bethune, B.: Mechanisms of wear of the metal surface during fretting corrosion of steel on polymers. Corros. Sci. 18(1), 3-13 (1978)

Hunter, S.C.: The Hertz problem for a rigid spherical indenter and a viscoelastic half-space. J. Mech. Phys. Solids 8(4), 219-234 (1960)

Lee, E.H.: Stress analysis in viscoelastic bodies. Q. Appl. Math. 13(2), 183-190 (1955)

Lee, E.H., Radok, J.R.M.: The contact problem for viscoelastic bodies. J. Appl. Mech. 27(3), 438-444 (1960)

Mao, X., Liu, W., Ni, Y., Popov, V.L.: Limiting shape of profile due to dual-mode fretting wear in contact with an elastomer. Pro Inst. Mech. Eng. C J. Mech. Eng. Sci. 230(9), 1417-1423 (2016)

Popov, V.L., Heß, M.: Method of dimensionality reduction in contact mechanics and friction. Springer, Heidelberg (2015). ISBN 978-3-642-53875-9

Radok, J.R.M.: Viscoelastic stress analysis. Q. Appl. Math. 15(2), 198-202 (1957)

Ting, T.C.T.: The contact stresses between a rigid indenter and a viscoelastic half-space. J. Appl. Mech. 33(4), 845-854 (1966)

Ting, T.C.T.: Contact problems in the linear theory of viscoelasticity. J. Appl. Mech. 35(2), 248-254 (1968)

Vandamme, M., Ulm, F.-J.: Viscoelastic solutions for conical indentation. Int. J. Solids Struct. 43(10), 3142-3165 (2006)

Open Access This chapter is licensed under the terms of the Creative Commons Attribution 4.0 International License (http://creativecommons.org/licenses/by/4.0/), which permits use, sharing, adaptation, distribution and reproduction in any medium or format, as long as you give appropriate credit to the original author(s) and the source, provide a link to the Creative Commons license and indicate if changes were made.

The images or other third party material in this chapter are included in the chapter's Creative Commons license, unless indicated otherwise in a credit line to the material. If material is not included in the chapter's Creative Commons license and your intended use is not permitted by statutory regulation or exceeds the permitted use, you will need to obtain permission directly from the copyright holder.

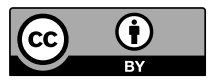

